\title{
Categoricity in Quasiminimal Pregeometry Classes
}

\author{
Levon Haykazyan*
}

October 1, 2018

\begin{abstract}
Quasiminimal pregeometry classes were introduces by Zilber 2005a to isolate the model theoretical core of several interesting examples. He proves that a quasiminimal pregeometry class satisfying an additional axiom, called excellence, is categorical in all uncountable cardinalities. Recently Bays et al. [2014] showed that excellence follows from the rest of axioms. In this paper we present a direct proof of the categoricity result without using excellence.
\end{abstract}

\section{Introduction}

Quasiminimal pregeometry class is a non elementary class of structures satisfying certain axioms. The notion was introduced by Zilber $|2005 \mathrm{a}|$ to give canonical axiomatisations of pseudo-exponential fields (in Zilber 2005b]) and other related analytic structures. The original definition of Zilber [2005a] had an additional axiom called excellence, which played a central role in establishing categoricity in cardinalities above $\aleph_{1}$. (And hence the original terminology of a quasiminimal excellent class.) The notion has evolved through works of Baldwin [2009] and Kirby [2010]. In practice checking that the excellence holds has been the most technically difficult part in applications of the categoricity theorem. Some of the original proofs of excellence had gaps, which have only recently been fixed.

Later Bays et al. [2014] showed that excellence is redundant in that it follows from the rest of axioms. In this paper we present a direct proof of categoricity result that bypasses excellence altogether. The main new idea

${ }^{*}$ This work was completed through support of the University of Oxford Clarendon Fund Scholarship. 
is to look at (partial) embeddings that preserve all $\mathcal{L}_{\omega_{1}, \omega}$ formulas possibly using infinitely many parameters. We call them $\sigma$-embeddings. Constructing $\sigma$-embeddings by a transfinite recursion presents additional challenges. Given an increasing chain $\left\langle f_{\beta}: \beta<\alpha\right\rangle$ of $\sigma$-embeddings, their union $f=\bigcup_{\beta<\alpha} f_{\beta}$ need not be a $\sigma$-embedding. The problem is that $f$ needs to preserve formulas over infinitely (countably) many parameters and if $\operatorname{cf}(\alpha)=\omega$ we cannot guarantee that all these parameters occur at an earlier stage. In case of quasiminimal pregeometry classes the axiom of $\aleph_{0}$-homogeneity over countable closed models provides a way around this problem in certain situations.

The rest of the paper is organised as follows. The next section fixes the notation and gives basic definitions. Section 3 establishes infinitary analogues of some well-known elementary properties in first order model theory. Then we prove the categoricity theorem and finish with some concluding remarks.

The author is grateful to Martin Bays, Jonathan Kirby and Boris Zilber for helpful discussions and comments.

\section{Background}

Let $\mathcal{L}$ be a finitary language, that is $\mathcal{L}$ has constant, functional and relation symbols of finite arity. For an infinite cardinal $\kappa$ the formulas of $\mathcal{L}_{\kappa, \omega}$ are inductively defined as follows.

- Atomic $\mathcal{L}$-formulas are $\mathcal{L}_{\kappa, \omega}$-formulas.

- If $\Phi$ is a set of $\mathcal{L}_{\kappa, \omega}$-formulas and $|\Phi|<\kappa$, then $\bigwedge_{\phi \in \Phi} \phi$ and $\bigvee_{\phi \in \Phi} \phi$ are $\mathcal{L}_{\kappa, \omega}$-formulas.

- If $\phi$ is an $\mathcal{L}_{\kappa, \omega}$-formula and $v$ is a variable, then $\neg \phi, \forall v \phi$ and $\exists v \phi$ are $\mathcal{L}_{\kappa, \omega}$-formulas.

In this notation the ordinary first-order language coincides with $\mathcal{L}_{\omega, \omega}$. Note that we do not require $\mathcal{L}_{\kappa, \omega}$-formulas to have finitely many free variables. However, every subformula of an $\mathcal{L}_{\kappa, \omega}$-sentence has finitely many free variables. If $M$ is an $\mathcal{L}$-structure, $\phi$ is an $\mathcal{L}_{\kappa, \omega}$-formula and $\theta$ is a variable assignment, then $M \models_{\theta} \phi$ is defined as usual. In particular

$$
M \models_{\theta} \bigwedge_{\phi \in \Phi} \phi \text { if and only if } M \models_{\theta} \phi \text { for all } \phi \in \Phi
$$

and

$$
M \models_{\theta} \bigvee_{\phi \in \Phi} \phi \text { if and only if } M \models_{\theta} \phi \text { for some } \phi \in \Phi \text {. }
$$


Two structures $M$ and $N$ are called equivalent in $\mathcal{L}_{\kappa, \omega}$ (in symbols $M \equiv_{\kappa, \omega} N$ ) if they satisfy the same $\mathcal{L}_{\kappa, \omega}$-sentences.

A formula of $\mathcal{L}_{\infty, \omega}$ is a formula of $\mathcal{L}_{\kappa, \omega}$ for some $\kappa$. The notation $M \equiv_{\infty, \omega}$ $N$ means that $M$ and $N$ satisfy the same $\mathcal{L}_{\infty, \omega}$-sentences.

Let $M, N$ be $\mathcal{L}$-structures and $f$ be a (partial) function from $M$ to $N$. (We use the notation $f: M \rightarrow N$ for partial functions). Then $f$ is called a (partial) embedding if it preserves quantifier-free formulas. Note that in particular $f$ preserves the formula $x=x$ and hence $f$ is injective. A bijective embedding is an isomorphism between $M$ and $N$. The function $f$ is called a (partial) elementary embedding if it preserves first-order formulas and a (partial) $\sigma$-embedding if it preserves all $\mathcal{L}_{\omega_{1}, \omega}$-formulas (in general using infinitely many parameters from the $\operatorname{dom}(f))$. In other words $f$ is a $\sigma$-embedding if $\langle M, m\rangle_{m \in \operatorname{dom}(f)} \equiv_{\omega_{1}, \omega}\langle N, f(m)\rangle_{m \in \operatorname{dom}(f)}$.

A back-and-forth system between $\mathcal{L}$-structures $M$ and $N$ is a nonempty collection $F$ of partial embeddings such that the following two conditions are satisfied:

- for all $f \in F$ and $a \in M$ there is $g \in F$ such that $f \subseteq g$ and $a \in \operatorname{dom}(g)$;

- for all $f \in F$ and $b \in N$ there is $g \in F$ such that $f \subseteq g$ and $b \in \operatorname{img}(g)$.

The following characterisation of $\mathcal{L}_{\infty, \omega}$-equivalence is due to Karp [1965].

Theorem 1. Let $M$ and $N$ be $\mathcal{L}$-structures. Then $M \equiv_{\infty, \omega} N$ if and only if there is a back and forth system between them.

Now we introduce the notion of a quasiminimal pregeometry class from Zilber [2005a]. Our definition follows closely that of Kirbv [2010]. For the definition of pregeometry the reader can consult Marker [2002].

Definition 2. Let $\mathcal{L}$ be a language. A quasiminimal pregeometry class is a class $\mathcal{C}$ of pairs $\left\langle H, \mathrm{cl}_{H}\right\rangle$ where $H$ is an $\mathcal{L}$-structure and $\mathrm{cl}_{H}: \mathcal{P}(H) \rightarrow \mathcal{P}(H)$ is a function satisfying the following conditions.

- Closure under isomorphisms If $\left\langle H, \mathrm{cl}_{H}\right\rangle \in \mathcal{C}, H^{\prime}$ is an $\mathcal{L}$-structure and $f: H \rightarrow H^{\prime}$ is an isomorphism, then $\left\langle H^{\prime}, \operatorname{cl}_{H^{\prime}}\right\rangle \in \mathcal{C}$, where $\operatorname{cl}_{H^{\prime}}$ is defined as $\operatorname{cl}_{H^{\prime}}\left(X^{\prime}\right)=f\left(\operatorname{cl}_{H}\left(f^{-1}\left(X^{\prime}\right)\right)\right)$ for $X^{\prime} \subseteq H^{\prime}$.

- Quantifier free theory

If $\left\langle H, \mathrm{cl}_{H}\right\rangle,\left\langle H^{\prime}, \mathrm{cl}_{H}^{\prime}\right\rangle \in \mathcal{C}$, then $H$ and $H^{\prime}$ satisfy the same quantifier free sentences. In other words the empty function is a partial embedding between any two structures. 
- Pregeometry

- For each $\left\langle H, \mathrm{cl}_{H}\right\rangle \in \mathcal{C}$ the function $\mathrm{cl}_{H}$ is a pregeometry on $H$ and the closure of any finite set is countable.

- If $\left\langle H, \mathrm{cl}_{H}\right\rangle \in \mathcal{C}$ and $X \subseteq H$, then $\mathrm{cl}_{H}(X)$ together with the restriction of $\mathrm{cl}_{H}$ is in $\mathcal{C}$.

- If $\left\langle H, \mathrm{cl}_{H}\right\rangle,\left\langle H^{\prime}, \mathrm{cl}_{H^{\prime}}\right\rangle \in \mathcal{C}, X \subseteq H, y \in H$ and $f: H \rightarrow H^{\prime}$ is a partial embedding defined on $X \cup\{y\}$, then $y \in \operatorname{cl}_{H}(X)$ if and only if $f(y) \in \mathrm{cl}_{H^{\prime}}(f(X))$.

- $\aleph_{0}$-homogeneity over countable closed models

Let $\left\langle H, \mathrm{cl}_{H}\right\rangle,\left\langle H^{\prime}, \mathrm{cl}_{H^{\prime}}\right\rangle \in \mathcal{C}$, subsets $G \subseteq H, G^{\prime} \subseteq H^{\prime}$ be countable closed or empty and $g: G \rightarrow G^{\prime}$ be an isomorphism.

- If $x \in H, x^{\prime} \in H^{\prime}$ are independent from $G$ and $G^{\prime}$ respectively, then $g \cup\left\{\left\langle x, x^{\prime}\right\rangle\right\}$ is a partial embedding.

- If $g \cup f: H \rightarrow H^{\prime}$ is a partial embedding, $X=\operatorname{dom}(f)$ is finite and $y \in \operatorname{cl}_{H}(X \cup G)$, then there is $y^{\prime} \in H^{\prime}$ such that $g \cup f \cup\left\{\left\langle y, y^{\prime}\right\rangle\right\}$ is a partial embedding.

To illustrate the definition we give some examples of quasiminimal pregeometry classes.

Example 3. The class of models of a strongly minimal first order theory together with algebraic closure is a quasiminimal pregeometry class, provided that the closure of the empty set is infinite. The later condition is needed to ensure that the closure of any subset of a given model is a model of the theory itself. This can be readily checked by Tarski-Vaught test, using the strong minimality of the theory.

Let the language $\mathcal{L}$ contain just one binary relation $E$. Consider the class of $\mathcal{L}$-structures, where $E$ is an equivalence relation and each equivalence class is countable. Define the closure of $X$ to be the set of elements equivalent to some $x \in X$. This class is a quasiminimal pregeometry class. It can be realised as the class of models of an $\mathcal{L}(Q)$-sentence.

Finally we mention mathematically interesting non elementary classes of pseudo-exponential fields of Zilber [2005b] and group covers of Zilber [2006].

A class satisfying the above conditions and an additional condition referred to as excellence, is called a quasiminimal excellent class in Zilber [2005a], Baldwin [2009], Kirby [2010]. It is shown in these works that any two structures in a quasiminimal excellent class of the same uncountable cardinality are isomorphic. As mentioned above Bays et al. [2014] showed that 
excellence follows from other conditions. The terminology of a quasiminimal pregeometry class comes from Bays et al. [2014], where the countable dimensional structure is called a quasiminimal pregeometry structure. Thus combining the results of Zilber [2005a] and Bays et al. [2014] we get that two structures in a quasiminimal pregeometry class of the same uncountable cardinality are isomorphic. In this paper we present a direct proof of this result.

\section{Properties of Structures in Quasiminimal Pregeometry Classes}

Fix a quasiminimal pregeometry class $\mathcal{C}$. The closure operator is often understood, so we will simply refer to $\mathcal{C}$ as a class of structures instead of a class of pairs. Given a structure $H \in \mathcal{C}$ and a substructure $G \subseteq H$ also in $\mathcal{C}$, we denote $G \preceq H$ the fact that $G$ is closed in $H$.

Proposition 4. Let $H, H^{\prime} \in \mathcal{C}, H \preceq H^{\prime}$ and $X \subseteq H$. Then $\operatorname{cl}_{H}(X)=$ $\operatorname{cl}_{H^{\prime}}(X)$.

Proof. Consider the identity embedding from $H$ to $H^{\prime}$. For $y \in H$ we have $y \in \mathrm{cl}_{H}(X)$ if and only if $y \in \mathrm{cl}_{H^{\prime}}(X)$. Hence $\mathrm{cl}_{H}(X)=\mathrm{cl}_{H^{\prime}}(X) \cap H$. But $\operatorname{cl}_{H^{\prime}}(X) \subseteq \operatorname{cl}_{H^{\prime}}(H)=H$. Hence $\operatorname{cl}_{H}(X)=\operatorname{cl}_{H^{\prime}}(X)$.

In view of this, we will drop the subscript from closure operator whenever no confusion arises. Let us prove some direct consequences of $\aleph_{0^{-}}$ homogeneity.

Proposition 5. Let $H, H^{\prime} \in \mathcal{C}$, subsets $G \subseteq H, G^{\prime} \subseteq H^{\prime}$ be countable closed or empty, $g: G \rightarrow G^{\prime}$ be an isomorphism and $g \cup f: H \rightarrow H^{\prime}$ be a partial embedding with $X=\operatorname{dom}(f), X^{\prime}=\operatorname{img}(f)$ finite.

- The mapping $g \cup f$ extends to an isomorphism $\hat{g}: \operatorname{cl}(X \cup G) \rightarrow \operatorname{cl}\left(X^{\prime} \cup\right.$ $\left.G^{\prime}\right)$.

- If $y \in H \backslash \operatorname{cl}(X \cup G)$ and $y^{\prime} \in H^{\prime} \backslash \operatorname{cl}\left(X^{\prime} \cup G^{\prime}\right)$, then $g \cup f \cup\left\{\left\langle y, y^{\prime}\right\rangle\right\}$ is a partial embedding.

Proof. For the first assertion note that by countable closure property both $\operatorname{cl}(X \cup G)$ and $\operatorname{cl}\left(X^{\prime} \cup G^{\prime}\right)$ are countable. Let $\left\langle a_{n}: n<\omega\right\rangle$ and $\left\langle b_{n}: n<\omega\right\rangle$ enumerate $\operatorname{cl}(X \cup G)$ and $\operatorname{cl}\left(X^{\prime} \cup G^{\prime}\right)$ respectively. Construct an increasing family $\left\langle f_{n}: n<\omega\right\rangle$ of finite mappings such that $g \cup f_{n}$ is a partial embedding as follows. Let $f_{0}=f$. For odd $n$ pick the least $m$ such that $a_{m}$ is not 
in the domain of $f_{n-1}$. By $\aleph_{0}$-homogeneity there is $b \in \operatorname{cl}\left(X^{\prime} \cup G^{\prime}\right)$ such that $g \cup f_{n-1} \cup\left\{\left\langle a_{m}, b\right\rangle\right\}$ is a partial embedding. Put $f_{n}=f_{n-1} \cup\left\{\left\langle a_{m}, b\right\rangle\right\}$. For even $n$ do the other way around. Then $\hat{g}=\bigcup_{n<\omega} f_{n}$ is an isomorphism between $\operatorname{cl}(X \cup G)$ and $\operatorname{cl}\left(X^{\prime} \cup G^{\prime}\right)$.

For the second assertion extend $g \cup f$ to an isomorphism $\hat{g}: \operatorname{cl}(X \cup G) \rightarrow$ $\operatorname{cl}\left(X^{\prime} \cup G^{\prime}\right)$. Now $\hat{g} \cup\left\{\left\langle y, y^{\prime}\right\rangle\right\}$ is a partial embedding by $\aleph_{0}$-homogeneity. Hence $g \cup f \cup\left\{\left\langle y, y^{\prime}\right\rangle\right\}$ is also a partial embedding.

Next we introduce $\sigma$-types and prove $\sigma$-saturation of uncountable structures in $\mathcal{C}$.

Definition 6. Let $H \in \mathcal{C}, A \subseteq H$ and $\bar{v}$ be a finite tuple of variables. A $\sigma$-type $p$ (in $H$ ) over $A$ in variables $\bar{v}$ is a set of $\mathcal{L}_{\omega_{1}, \omega}$-formulas with parameters from $A$ and free variables among $\bar{v}$ such that every countable subset is consistent with $H$. That is for every countable $\Phi \subseteq p$ we have $H \models \exists \bar{v} \bigwedge_{\phi \in \Phi} \phi(\bar{v})$.

If the length of tuple $\bar{v}$ is $n$, then we call $p$ an $n$-type. We can think semantically of $\sigma$-types as a family of $\mathcal{L}_{\omega_{1}, \omega}$-definable subsets such that each countable subfamily has a nonempty intersection. A $\sigma$-type $p$ is complete if for every $\mathcal{L}_{\omega_{1}, \omega}$-formula $\phi(\bar{v})$ we have either $\phi \in p$ of $\neg \phi \in p$. This corresponds to a $\sigma$-complete ultrafilter on the $\sigma$-algebra of $\mathcal{L}_{\omega_{1}, \omega}$-definable subsets. A $\sigma$-type $p$ is called isolated if there is a consistent $\mathcal{L}_{\omega_{1}, \omega}$-formula $\psi(\bar{x})$ such that $H \models \forall \bar{x}(\psi(\bar{x}) \rightarrow \phi(\bar{x}))$ for all $\phi \in p$. A $\sigma$-type $p$ is realised in $H$ if $\bigcap_{\phi \in p} \phi\left(H^{n}\right) \neq \emptyset$. Clearly each isolated $\sigma$-type is realised. Since $\mathcal{L}_{\omega_{1}, \omega^{-}}$ definable sets are closed under countable intersections, we have the following

Proposition 7. If a $\sigma$-type contains a formula defining a countable set, then it is isolated and hence realised.

Now let us study $\sigma$-types in quasiminimal pregeometry structures.

Proposition 8. Let $H \in \mathcal{C}$ and $X \subseteq H$ be countable. Let $a, b \in H \backslash \operatorname{cl}(X)$. Then $a$ and $b$ realise the same $\sigma$-type over $X$.

Proof. Let $G=\operatorname{cl}(X)$ and $g_{0}=\operatorname{id}_{G} \cup\{\langle a, b\rangle\}$. By $\aleph_{0}$-homogeneity $g_{0}$ is an embedding. Now consider the collection $F$ of finite extensions of $g_{0}$ that are embeddings. It is not empty as $g_{0} \in F$. We claim that $F$ is a back-andforth system. Indeed if $g \in F$ and $y \in H$, then $g=\operatorname{id}_{G} \cup f$, where $f$ has finite domain. If $y \in \operatorname{cl}(\operatorname{dom}(g))$, then use $\aleph_{0}$-homogeneity to extend $g$ to $y$. Otherwise there is $y^{\prime} \in H \backslash \operatorname{cl}(\operatorname{img}(g))$. By Proposition 5 the map $g \cup\left\{\left\langle y, y^{\prime}\right\rangle\right\}$ is an embedding. Similarly, we can extend $g$ to an embedding with $y$ in the image. 
Now expand the language by adding constant symbols $c_{g}$ for each $g \in G$ and an additional constant $c$. Let $H_{1}$ be an expansion of $H$ by interpreting $c_{g}$ by $g$ and $c$ by $a$. Similarly let $H_{2}$ be an expansion of $H$ by interpreting $c_{g}$ by $g$ and $c$ by $b$. By the above there is a back-and-forth system between $H_{1}$ and $H_{2}$. Hence $H_{1} \equiv_{\infty, \omega} H_{2}$. In particular $H_{1} \equiv_{\omega_{1}, \omega} H_{2}$. It follows that every sentence using parameters from $G$ that is true on $a$ is also true on $b$. Hence $a$ and $b$ realise the same $\sigma$-type over $G$. Since $X \subseteq G$, elements $a$ and $b$ also realise the same $\sigma$-type over $X$.

The next corollary establishes the analogy between quasiminimal pregeometry structures and minimality in first-order context. It is also the motivation behind the term quasiminimality.

Corollary 9. Let $H \in \mathcal{C}$ and $\phi(v)$ be an $\mathcal{L}_{\omega_{1}, \omega}$ formula (possibly using parameters). Then $\phi(H)$ is either countable or cocountable.

Proof. Suppose otherwise. Let $\bar{c}$ be the parameters used in $\phi$. Then $\bar{c}$ is countable. Since both $\phi(H)$ and $\neg \phi(H)$ are uncountable, there are $a \in$ $\phi(H) \backslash \operatorname{cl}(\bar{c})$ and $b \in \neg \phi(H) \backslash \operatorname{cl}(\bar{c})$. This contradicts the fact that $a$ and $b$ realise the same $\sigma$-type over $\bar{c}$.

And now we establish the analogy between the closure in quasiminimal pregeometry classes and algebraic closure.

Corollary 10. Let $H \in \mathcal{C}$ be uncountable, and $X \subseteq H$ be a countable subset. Then $y \in \operatorname{cl}(X)$ if and only if it satisfies an $\mathcal{L}_{\omega_{1}, \omega}$-formula that has countably many solutions.

Proof. Assume that $y$ satisfies $\phi(v)$ that has countably many solutions. Since $\operatorname{cl}(X)$ is countable, there is $y^{\prime} \in \neg \phi(H) \backslash \operatorname{cl}(X)$. Now $y$ and $y^{\prime}$ do not satisfy the same type over $X$. Hence $y \in \operatorname{cl}(X)$.

Conversely assume that $y \in \operatorname{cl}(X)$. Pick $y^{\prime} \in H \backslash \operatorname{cl}(X)$. Since the closure is determined by the language, the map $\operatorname{id}_{X} \cup\left\{\left\langle y, y^{\prime}\right\rangle\right\}$ is not an embedding. Hence there is a quantifier-free formula $\phi(v)$ over $X$ satisfied by $y$ but not $y^{\prime}$. Now $\neg \phi(H)$ cannot by countable (as it implies that $y^{\prime} \in \operatorname{cl}(X)$ ). Hence $\phi(H)$ is countable.

Next we introduce the infinitary analogue of saturation and prove this property for uncountable structures in quasiminimal pregeometry classes.

Definition 11. A structure $H$ is called $\sigma$-saturated if for every $X \subset H$ with $|X|<|H|$ every $\sigma$-type over $X$ is realised in $H$.

Proposition 12. Let $H \in \mathcal{C}$ be uncountable. Then $H$ is $\sigma$-saturated. 
Proof. Let $X \subset H$ be a subset with $|X|<|H|$ and let $p$ be a $\sigma$-type over $X$ in $n$ variables. We prove by induction on $n$ that $p$ is realised in $H$.

Let $n=1$. Put $G=\operatorname{cl}(X)$. Then $|G|=|X|+\aleph_{0}<|H|$. So there is $y \in H \backslash G$. If $y$ realises $p$, then we are done. So assume the opposite. Then there is a formula $\phi(v) \in p$ such that $H \models \neg \phi(y)$. Now since $y \notin \mathrm{cl}(X)$, we have $\neg \phi(H)$ is uncountable. Hence $\phi(H)$ is countable. But then $p$ is isolated and hence realised in $H$.

Assume the hypothesis for $n$. Let $p$ be an $n+1$-type. As before let $G=$ $\operatorname{cl}(X)$. We claim that there is $x \in H$ such that $q_{x}=\{\phi(\bar{v}, x): \phi(\bar{v}, w) \in p\}$ is a $\sigma$-type. Assume the opposite. Then for every $x$ there is a countable subset $p_{x} \subset p$ such that

$$
H \models \neg \exists \bar{v} \bigwedge_{\phi \in p_{x}} \phi(\bar{v}, x) .
$$

Pick $y \in H \backslash G$ and let $Y$ be the set of parameters used in formulas of $p_{y}$. Then $Y \subseteq X$ is countable and $y \notin \operatorname{cl}(Y)$. But since every two elements outside $\operatorname{cl}(Y)$ realise the same type over $Y$, for every $z \in H \backslash \operatorname{cl}(Y)$ we have

$$
H \models \neg \exists \bar{v} \bigwedge_{\phi \in p_{y}} \phi(\bar{v}, z) .
$$

Now let $W=p_{y} \cup \bigcup_{x \in \operatorname{cl}(Y)} p_{x}$. Then $W$ is countable and we have that

$$
H \models \neg \exists w \exists \bar{v} \bigwedge_{\phi \in W} \phi(\bar{v}, w) .
$$

This contradicts the fact that $p$ is a $\sigma$-type. Thus for some $x \in H$ we have that $q_{x}=\{\phi(\bar{v}, x): \phi(\bar{v}, w) \in p\}$ is a $\sigma$-type. By induction hypothesis $q_{x}$ is realised in $H$ and hence $p$ is also realised in $H$.

\section{The Categoricity Theorem}

In this section we prove that any two structures of the same uncountable cardinality in a quasiminimal pregeometry class are isomorphic. Let $H, H^{\prime} \in \mathcal{C}$ be of the same uncountable cardinality. Since we can construct a backand-forth system between $H$ and $H^{\prime}$, we have that $H \equiv_{\omega_{1}, \omega} H^{\prime}$. In other words the empty embedding is a $\sigma$-embedding. In analogy with first-order case we would like to extend a partial $\sigma$-embedding to map $H$ onto $H^{\prime}$. By $\sigma$-saturation we can extend any $\sigma$-embedding to any one element (and recursively to any finite number of elements). At limit stages however, we need to take unions. But the union of $\sigma$-embeddings may not be a $\sigma$-embedding. However, the union of $\sigma$-embeddings is always an embedding and in some cases this is sufficient to get a $\sigma$-embedding. 
Proposition 13. Let $H, H^{\prime} \in \mathcal{C}$ be uncountable, subsets $G \subset H, G^{\prime} \subset H^{\prime}$ be countable closed and let $g: G \rightarrow G^{\prime}$ be an isomorphism. Then $g$ is a partial $\sigma$-embedding between $H$ and $H^{\prime}$.

Proof. By $\aleph_{0}$-homogeneity and Proposition 5 the set of embeddings between $H$ and $H^{\prime}$ that are finite extensions of $g$ is a back-and-forth system. Hence if we add constant symbols for $G$ in $H$ and for $G^{\prime}$ in $H^{\prime}$ the resulting structures will be $\mathcal{L}_{\omega_{1}, \omega}$-equivalent. Therefore $g$ is a $\sigma$-embedding.

We can use this result to extend a $\sigma$-embeddings to the closure of its domain provided the later is countable.

Proposition 14. Let $H, H^{\prime} \in \mathcal{C}$ be uncountable and let $g: H \rightarrow H^{\prime}$ be a partial $\sigma$-embedding with $X=\operatorname{dom}(g), X^{\prime}=\operatorname{img}(g)$ countable. Then $g$ extends to a $\sigma$-embedding $\hat{g}: H \rightarrow H^{\prime}$ with $\operatorname{dom}(\hat{g})=\operatorname{cl}(X)$ and $\operatorname{img}(\hat{g})=$ $\operatorname{cl}\left(X^{\prime}\right)$.

Proof. Let $\operatorname{cl}(X)=\left\{a_{n}: n<\omega\right\}$ and $\operatorname{cl}\left(X^{\prime}\right)=\left\{a_{n}^{\prime}: n<\omega\right\}$. Construct an increasing sequence $f_{0} \subseteq f_{1} \subseteq f_{2} \ldots$ of $\sigma$-embeddings as follows. Let $f_{0}=g$. For even $n$, pick the least $m$ not in the domain of $f_{n}$. Let $p$ be the $\sigma$-type of $a_{m}$ over $\operatorname{dom}\left(f_{n}\right)$. Consider the $\sigma$-type $p^{\prime}=\left\{\phi\left(x, f_{n}(\bar{b})\right): \phi(x, \bar{b}) \in p\right\}$. The set $p^{\prime}$ is a $\sigma$-type as $f_{n}$ is a $\sigma$-embedding. By $\sigma$-saturation of $H^{\prime}$, the type $p^{\prime}$ is realised by some $c \in H^{\prime}$. Let $f_{n+1}=f_{n} \cup\left\{\left\langle a_{m}, c\right\rangle\right\}$. For odd $n$ go the other direction.

Now take $\hat{g}=\bigcup_{n<\omega} f_{n}$. Then $\hat{g}$ is an embedding between countable closed set $\mathrm{cl}(X)$ and $\mathrm{cl}\left(X^{\prime}\right)$. By Proposition 13 the embedding $\hat{g}$ is a $\sigma$ embedding.

In particular every countable embedding that extends to the closure of its domain must be a $\sigma$-embedding.

Corollary 15. Let $H, H^{\prime} \in \mathcal{C}$ be uncountable, $G \subset H, G^{\prime} \subset H^{\prime}$ be countable closed or empty, $g: G \rightarrow G^{\prime}$ an isomorphism, $a \in H \backslash G$ and $a^{\prime} \in H^{\prime} \backslash G^{\prime}$. Then $g \cup\left\{\left\langle a, a^{\prime}\right\rangle\right\}$ is a $\sigma$-embedding.

Now we can prove the main result of this paper. The main difference between our approach and the existing literature is the focus on $\sigma$-embeddings. The existing proofs of categoricity start with an ordinary embedding (i.e. a function that preserves quantifier free formulas) and extend it to an isomorphism between two structures of the same cardinality. At certain stages of the construction one needs to extend an embedding with domain of a special form to its closure. The condition of excellence is precisely the statement that this is possible. However, if we have a $\sigma$-embedding at hand, then we can always extend it to the closure of its domain by Proposition 14 (provided the domain is countable). This is where we bypass the need for excellence. 
Theorem 16. Let $H, H^{\prime} \in \mathcal{C}$ be uncountable, let $B, B^{\prime}$ be bases of $H, H^{\prime}$ respectively and let $g: B \rightarrow B^{\prime}$ be a bijection. Then $g$ extends to an isomorphism $\hat{g}: H \rightarrow H^{\prime}$.

Proof. Let $B=\left\{b_{\alpha}: \alpha<\kappa\right\}, B^{\prime}=\left\{b_{\alpha}^{\prime}: \alpha<\kappa\right\}$ and $g\left(b_{\alpha}\right)=b_{\alpha}^{\prime}$. For $n<\omega$, let $G_{n}=\operatorname{cl}\left(\left\{b_{m}: n \leq m<\omega\right\}\right)$ and $G_{n}^{\prime}=\operatorname{cl}\left(\left\{b_{m}^{\prime}: n \leq m<\omega\right\}\right)$. By $\aleph_{0}$-homogeneity there is an isomorphism $f_{0}: G_{0} \rightarrow G_{0}^{\prime}$ extending $g$. By Proposition 13 the embedding $f_{0}$ is a $\sigma$-embedding.

For each finite subset $X \subset B$ we construct a number $n_{X}$ and a surjective $\sigma$-embedding $f_{X}: \operatorname{cl}\left(G_{n_{X}} X\right) \rightarrow \operatorname{cl}\left(G_{n_{X}}^{\prime} X^{\prime}\right)$ that extends $g$ and satisfies the following condition: whenever $X \subseteq Y$, we have $n_{X} \leq n_{Y}$ and $\left.f_{X}\right|_{\operatorname{cl}\left(G_{n_{Y}} X\right)}=$ $\left.f_{Y}\right|_{\mathrm{cl}\left(G_{n_{Y}} X\right)}$.

Assume that we have constructed such embeddings. Define $\hat{g}: H \rightarrow H^{\prime}$ as follows. For every $x \in H$, we have $x \in \operatorname{cl}(X)$ for some finite $X \subset B$. Define $\hat{g}(x)=f_{X}(x)$. By the assumption on the embeddings, the result does not depend on the choice of $X$. Now $\hat{g}$ is surjective. Indeed for $x^{\prime} \in H^{\prime}$ pick finite $X^{\prime} \subset B^{\prime}$ such that $x^{\prime} \in \operatorname{cl}\left(X^{\prime}\right)$. Let $X=g^{-1}\left(X^{\prime}\right)$. Then $x^{\prime} \in \operatorname{img}\left(f_{X}\right)$. Since $f_{X}$ is an embedding $f_{X}^{-1}\left(x^{\prime}\right) \in \operatorname{cl}(X)$. Hence $\hat{g}\left(f_{X}^{-1}\left(x^{\prime}\right)\right)=x^{\prime}$. Also if $\bar{x} \in H$ is a finite tuple, choose $X \subset B$ a finite set such that $\bar{x} \in \operatorname{cl}(X)$. Then $\hat{g}(\bar{x})=f_{X}(\bar{x})$, preserves quantifier free formulas. Thus $\hat{g}$ is an isomorphism.

We now proceed to the construction of $\sigma$-embeddings $f_{X}$ by a wellfounded induction on partial order of subsets of $B$. Our construction is a modification of that of Baldwin [2009], Kirby [2010]. Take $n_{\emptyset}=0$ and $f_{\emptyset}=f_{0}$. If $X=\left\{b_{\alpha}\right\}$ is a singleton do the following. If $\alpha<\omega$, then take $n_{X}=\alpha+1$, otherwise take $n_{X}=0$. Then $\left.f_{0}\right|_{G_{n}}: G_{n_{X}} \rightarrow G_{n_{X}}^{\prime}$ is an isomorphism. By Corollary 15, the map $f_{0} \cup\left\{\left\langle b_{\alpha}, b_{\alpha}^{\prime}\right\rangle\right\}$ is a $\sigma$-embedding. So by Proposition 13 it extends to an isomorphism $f_{X}: \operatorname{cl}\left(G_{n_{X}} X\right) \rightarrow \operatorname{cl}\left(G_{n_{X}}^{\prime} X^{\prime}\right)$.

Let $X=\left\{x_{1}, \ldots, x_{l}\right\}, l>1$ and we have already constructed $n_{Y}$ and $f_{Y}$ for every $Y \subsetneq X$. Let $n=\max \left\{n_{Y}: Y \subsetneq X\right\}$. Then each $g_{Y}=\left.f_{Y}\right|_{\mathrm{cl}\left(G_{n} Y\right)}$ is a $\sigma$-embedding of $\operatorname{cl}\left(G_{n} Y\right)$ onto $\operatorname{cl}\left(G_{n}^{\prime} Y^{\prime}\right)$, where $Y^{\prime}=g(Y)$. Now if $x \in$ $\operatorname{dom}\left(g_{Y_{1}}\right) \cap \operatorname{dom}\left(g_{Y_{2}}\right)$ for $Y_{1}, Y_{2} \subsetneq X$, then $x \in \operatorname{dom}\left(g_{Y_{1} \cap Y_{2}}\right)$. Thus if we define $g_{X}=\bigcup_{Y \subsetneq X} g_{Y}$, then $g_{X}$ is a well defined function.

Let $Y_{i}=X \backslash\left\{x_{i}\right\}$ and $h_{k}=\bigcup_{i=1}^{k} g_{Y_{i}}$. So $g_{X}=h_{l}$. Then $h_{k}$ is a mapping from $\bigcup_{i=1}^{k} \operatorname{cl}\left(G_{n} Y_{i}\right)$ onto $\bigcup_{i=1}^{k} \operatorname{cl}\left(G_{n}^{\prime} Y_{i}^{\prime}\right)$.

Claim. For each $k=1, \ldots, l$ there is $m_{k} \geq n$ such that $\left.h_{k}\right|_{\bigcup_{i=1}^{k} \operatorname{cl}\left(G_{m_{k}} Y_{i}\right)}$ is a $\sigma$-embedding.

Proof. By induction on $k$. For $k=1$, we have that $h_{k}=g_{Y_{1}}$, hence we can just take $m_{k}=n$.

Assume the hypothesis for $k-1$. Then there is $m=m_{k-1} \geq n$ such that $\left.h_{k-1}\right|_{\bigcup_{i=1}^{k-1} \operatorname{cl}\left(G_{m} Y_{i}\right)}$ is a $\sigma$-embedding. Take $m_{k}=m+1$. Let $C_{k-1}=$ 
$\bigcup_{i=1}^{k-1} \operatorname{cl}\left(G_{m+1} Y_{i}\right)$. We should show that $\left.h_{k}\right|_{C_{k-1} \cup \mathrm{cl}\left(G_{m+1} Y_{k}\right)}$ is a $\sigma$-embedding. Let $\bar{a} \in C_{k-1}$ and $\bar{b} \in \operatorname{cl}\left(G_{m+1} Y_{k}\right)$ be (possibly infinite) tuples. Let $\theta$ be an automorphism of $\operatorname{cl}\left(G_{m} X\right)$ that fixes $\operatorname{cl}\left(G_{m+1} Y_{k}\right)$ and swaps $x_{k}$ with $b_{m}$

Let $e$ be a $\sigma$-embedding from $\operatorname{cl}\left(G_{m} X\right)$ onto $\operatorname{cl}\left(G_{m}^{\prime} X^{\prime}\right)$ extending the $\sigma$ embedding $\left.h_{k-1}\right|_{\bigcup_{i=1}^{k-1} \operatorname{cl}\left(G_{m} Y_{i}\right)}$. Let $\tau=e \theta^{-1} e^{-1} g_{Y_{k}} \theta$. The automorphism $\theta$ takes $C_{k-1} \cup \operatorname{cl}\left(G_{m+1} Y_{k}\right)$ to $\operatorname{cl}\left(G_{m} Y_{k}\right)$ and $g_{Y_{k}}$ is defined on it (as $m \geq n$ ). Hence $\tau$ is well defined. All these maps preserve $\mathcal{L}_{\omega_{1}, \omega}$ formulas. Hence so does $\tau$.

Let $Y_{i k}=Y_{i} \cap Y_{k}=X \backslash\left\{x_{i}, x_{k}\right\}$. Since $\bar{a} \in C_{k-1}=\bigcup_{i=1}^{k-1} \operatorname{cl}\left(G_{m+1} Y_{i}\right)$, we have that

$$
\theta(\bar{a}) \in \bigcup_{i=1}^{k-1} \operatorname{cl}\left(G_{m} Y_{i k}\right) .
$$

We have that $g_{Y_{k}}$ agrees with $g_{Y_{i}}$ on $\operatorname{cl}\left(G_{m} Y_{i k}\right)$. Hence $g_{Y_{k}}$ agrees with $h_{k-1}$ on $\bigcup_{i=1}^{k-1} \operatorname{cl}\left(G_{m} Y_{i k}\right)$. Also $e$ agrees with $h_{k-1}$ on $\bigcup_{i=1}^{k-1} \operatorname{cl}\left(G_{m} Y_{i}\right)$ and $h_{k}$ extends $h_{k-1}$. Thus

$$
\tau(\bar{a})=e \theta^{-1} e^{-1} g_{Y_{k}} \theta(\bar{a})=h_{k-1} \theta^{-1} h_{k-1}^{-1} h_{k-1} \theta(\bar{a})=h_{k-1}(\bar{a})=h_{k}(\bar{a}) .
$$

The tuple $\bar{b} \in \operatorname{cl}\left(G_{m+1} Y_{k}\right)$. Hence it is fixed by $\theta$. The embeddings $g_{Y_{k}}$ and $e$ preserve closure, hence $e^{-1} g_{Y_{k}}(b) \in \operatorname{cl}\left(G_{m+1} Y_{k}\right)$ is fixed by $\theta^{-1}$. Hence

$$
\tau(\bar{b})=e \theta^{-1} e^{-1} g_{Y_{k}} \theta(\bar{b})=e e^{-1} g_{Y_{k}}(\bar{b})=g_{Y_{k}}(\bar{b})=h_{k}(\bar{b}) .
$$

Thus if $\phi$ is any $\mathcal{L}_{\omega_{1}, \omega}$-formula, then

$$
H \models \phi(\bar{a}, \bar{b}) \Longleftrightarrow H^{\prime} \models \phi(\tau(\bar{a}), \tau(\bar{b})) \Longleftrightarrow H^{\prime} \models \phi\left(h_{k}(\bar{a}), h_{k}(\bar{b})\right) .
$$

Thus $h_{k}$ is a $\sigma$-embedding on $\bigcup_{i=1}^{k} \operatorname{cl}\left(G_{m+1} Y_{i}\right)$.

Thus there is some $r$ such that $g_{X}=h_{l}$ is a $\sigma$-embedding on $\bigcup_{i=1}^{l} \operatorname{cl}\left(G_{r} Y_{i}\right)$. Put $n_{X}=r$. Then $\left.h_{l}\right|_{\bigcup_{i=1}^{l} \operatorname{cl}\left(G_{r} Y_{i}\right)}$ extends to a $\sigma$-embedding $f_{X}$ from the closure of the domain $\operatorname{cl}\left(\bigcup_{i=1}^{l} \operatorname{cl}\left(G_{r} Y_{i}\right)\right)=\operatorname{cl}\left(G_{r} X\right)$ onto $\operatorname{cl}\left(G_{r}^{\prime} X^{\prime}\right)$ by Proposition 14 .

\section{Concluding Remarks}

Ever since its introduction by Shelah, excellence has been the key notion for categoricity in non-elementary classes. What our methods show is that in some very natural mathematical examples one can use infinitary logic instead. The fact that $\sigma$-embeddings and associated infinitary notions occur in natural mathematical contexts is remarkable in itself. This opens up a possibility of a broader use of infinitary logic both in elementary and nonelementary setting. 


\section{References}

John Baldwin. Categoricity. American Mathematical Society, 2009.

Martin Bays, Bradd Hart, Tapani Hyttinen, Meeri Kesälä, and Jonathan Kirby. Quasiminimal structures and excellence. Bulletin of London Mathematical Society, 46:155-163, 2014.

Carol Karp. Finite-quantifier equivalence. In John Addison, Leon Henkin, and Alfred Tarski, editors, The Theory of Models : Proceedings of the 1963 International Symposium at Berkeley. North-Holland, 1965.

Jonathan Kirby. On quasiminimal excellent classes. The Journal of Symbolic Logic, 75(2):551 - 564, 2010.

David Marker. Model Theory: An Introduction. Springer-Verlag New York, Inc., 2002.

Boris Zilber. A categoricity theorem for quasi-minimal excellent classes. In Andreas Blass and Yi Zhang, editors, Logic and its Applications. American Mathematical Society, 2005a.

Boris Zilber. Pseudo-exponentiation on algebraically closed fields of characteristic zero. Annals of Pure and Applied Logic, 132:67-95, 2005b.

Boris Zilber. Covers of the multiplicative group of an algebraically closed field of characteristic zero. Journal of the London Mathematical Society, 74(1):41-58, 2006. 